\title{
Ventajas del análisis Wavelet sobre el análisis de Fourier para la interpretación del ruido electroquímico
}

\author{
L. Espada*, M. Sanjurjo*, S. Urréjola*, F. Bouzada*, G. Rey* y A. Sánchez*
}

Resumen Por su sencillez y bajo coste frente a otros tipos de metodologías, la medida e interpretación del Ruido Electroquímico, REQ, se está consolidando como uno de los métodos de análisis más utilizados para la interpretación del fenómeno de la corrosión. Al ser una técnica en desarrollo, no existen todavía metodologías estandarizadas de tratamiento de los datos obtenidos en los experimentos. Hasta la fecha, se suelen utilizar el análisis estadístico y el análisis de Fourier para establecer parámetros que puedan caracterizar a los registros de ruido electroquímico de potencial e intensidad. En este trabajo se introduce una nueva metodología basada en el análisis Wavelet que presenta las ventajas, respecto del análisis de Fourier, de poder distinguir variaciones periódicas y no periódicas de la potencia de la señal tanto en el dominio del tiempo, como en el de la frecuencia, y no sólo en el dominio de la frecuencia como ocurre en el caso del análisis de Fourier.

Palabras clave Ruido electroquímico. Transformada wavelet. Análisis de Fourier. Corrosión.

\section{Advantages of Wavelet analysis compared to Fourier analysis for the interpretation of electrochemical noise}

\begin{abstract}
Given its simplicity and low cost compared to other types of methodologies, the measurement and interpretation of Electrochemical Noise, is consolidating itself as one of the analysis methods most frequently used for the interpretation of corrosion. As the technique is still evolving, standard treatment methodologies for data retrieved in experiments do not exist yet. To date, statistical analysis and the Fourier analysis are commonly used in order to establish the parameters that may characterize the recordings of potential and current electrochemical noise. This study introduces a new methodology based on wavelet analysis and presents its advantages with regards to the Fourier analysis in distinguishing periodical and non-periodical variations in the signal power in time and frequency, as opposed to the Fourier analysis that only considerers the frequency.
\end{abstract}

Keywords Electrochemical noise. Wavelet transform. Fourier analysis. Corrosion.

\section{INTRODUCCIÓN}

Los procesos físicos pueden ser clasificados según su naturaleza en determinísticos y no determinísticos, los primeros pueden dividirse a su vez en periódicos y no periódicos, mientras que en el caso de los fenómenos no determínisticos la subdivisión es en estacionarios y no estacionarios. Cada una de estas fenomenologías conlleva un tratamiento matemático específico. Los registros que se obtienen al medir las fluctuaciones espontáneas de potencial e intensidad que se producen cuando un electrodo se corroe libremente, se denominan Ruido Electroquímico, REQ ${ }^{[1]}$. El origen y el mecanismo que rigen los procesos que lo generan no están del todo claros. En la bibliografía ${ }^{[2]}$, distintos autores presentan este fenómeno como la manifestación de la superposición de los procesos anódicos y catódicos que coexisten en las interfases electrodo disolución. Este tipo de registros corresponde a un fenómeno aleatorio, no determinístico, no estacionario, cuyo campo propio de resolución es la teoría del caos y, en concreto, el análisis fractal. Sin embargo, dada la complejidad del análisis matemático

$\left({ }^{*}\right)$ Departamento de Ingeniería Química. E.T.S.E.I. e M. Universidade de Vigo.

${ }^{* *}$ Departamento de Ciencia de los Materiales. ET.S.E.I. e M. Universidade de Vigo Lagoas-Marcosende. 36200. VIGO (España). (SPAIN). 
por medio de este tipo de técnicas, la mayor parte de los autores que aplican el REQ al estudio de los procesos de corrosión optan en sus trabajos por utilizar otro tipo de metodología, como pudiera ser el análisis espectral, más propio de los datos determinísticos periódicos, o bien el análisis estadístico tradicional, cuyo campo específico de aplicación sería el de los procesos no determinísticos estacionarios.

Por otro lado, el análisis wavelet se está convirtiendo en una herramienta común para el estudio de las variaciones localizadas de la potencia dentro de una serie o registro temporal, como son los datos que se obtienen por medio de experimentos de ruido electroquímico. Mediante la descomposición de una serie temporal en un espacio tiempo-frecuencia, se pueden observar tanto modos dominantes de variabilidad de la señal (frecuencias), como la forma en que esos modos varían a su vez en el tiempo. La transformada Wavelet se ha usado ya con éxito en numerosos estudios en geofísica, electrónica y otros campos de la ciencia ${ }^{[3-5]}$ en sustitución del tradicional análisis de Fourier. Una descripción completa de aplicaciones de esta técnica puede encontrarse en la ref. ${ }^{[6]}$, mientras que su fundamento teórico lo estableció Daubechies ${ }^{[7]}$.

El propósito de este trabajo es introducir el análisis Wavelet al tratamiento de los registros de ruido electroquímico con el fin de establecer las bases de una herramienta de fácil uso para el análisis Wavelet. Para ello, se utilizarán registros reales de ruido electroquímico medidos en aluminio puro expuesto a tres diferentes medios en los que el metal sufre distintos tipos de corrosión. Además, se comparan los resultados obtenidos con los que se extraen del análisis de Fourier de los mismos registros.

\section{PARTE EXPERIMENTAL}

Para la obtención de datos de ruido electroquímico se utilizó un esquema de medida como el que se refleja en la figura 1. En este tipo de montaje experimental, habitual en muchos estudios de ruido electroquímico, se usa un sistema de tres electrodos. Tres cilindros de aluminio de pureza mayor del $99,9 \%$ y diámetro $8 \mathrm{~mm}$, embutidos en una resina orgánica de fraguado en frío se conectan a los aparatos de medida. Entre dos de ellos se miden las oscilaciones de potencial mediante un voltímetro digital y, entre uno de los anteriores y el tercero, el paso de corriente eléctrica (fluctuaciones de intensidad), mediante un amperímetro de resistencia cero. Para garantizar la minimización del ruido ex-

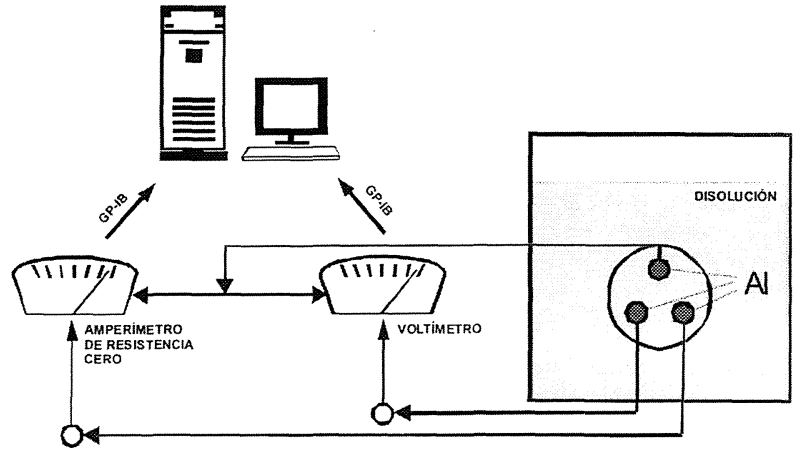

Figura 1. Esquema de medida.

Figure 1. Data acquisition scheme.

terior todo el sistema se mantuvo, durante el tiempo que duraron los experimentos, dentro de una caja de Faraday, llevándose las señales a los aparatos de medida mediante cable apantallado. Los registros de potencial e intensidad se realizaron en continuo durante cuarenta y ocho horas, agrupándose los datos en ficheros de 2.048 puntos de longitud. La frecuencia de muestreo fue de $2 \mathrm{~Hz}$, lo que significa que cada fichero abarca un período de $1.024 \mathrm{~s}$ de exposición. La longitud de los registros se toma como potencia de dos y la frecuencia de muestreo como $2 \mathrm{~Hz}$ para minimizar los errores numéricos derivados de la aplicación de la transformada discreta de Fourier ${ }^{[8]}$.

Como electrólitos se emplearon tres disoluciones tipo en las que se conoce el estado de corrosión del aluminio. Las composiciones de los electrólitos, así como el estado de corrosión del aluminio en dichos medios se resume en la tabla I.

\section{DISCUSIÓN}

En las figuras 2a, 2b, 3a, 3b, 4a y $4 \mathrm{~b}$ se encuentran los registros de potencial e intensidad del aluminio en cada una de las disoluciones estudiadas durante la duodécima hora de exposición, tiempo suficiente para que el sistema haya alcanzado un estado estacionario.

Tabla I. Disoluciones empleadas en los experimentos y tipos de corrosión que estas provocan en el aluminio

Table I. Solutions used in the experiments and types of corrosion caused to the aluminium

\begin{tabular}{cc}
\hline Disolución & Tipo de corrosión \\
\hline Agua destilada & pasivación \\
$\mathrm{NaCl}(3,5 \%)$ & picaduras \\
$\mathrm{NaOH}(1 \mathrm{M})$ & corrosión generalizada \\
\hline
\end{tabular}


Ventajas del análisis Wavelet sobre el análisis de Fourier para la interpretación del ruido electroquímico L. Espada, M. Sanjurjo, S. Urréjola, F. Bouzada, G. Rey y A. Sánchez
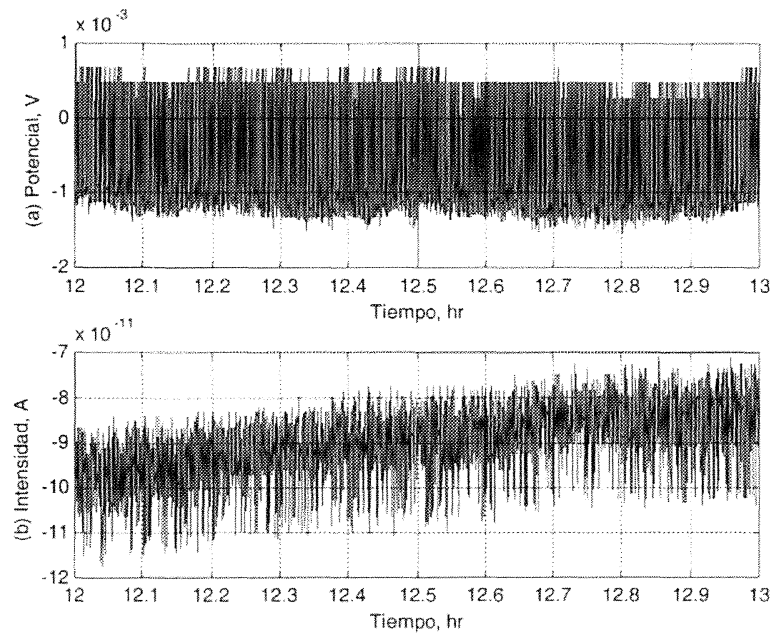

Figura 2. Registros de ruido electroquímico de potencial (a) e intensidad (b) del aluminio en agua destilada (pasivación) después de $12 \mathrm{~h}$ de exposición.

Figure 2. Electrochemical noise measurements for potential (a) and current (b) for aluminium in distilled water (passivation) after $12 \mathrm{~h}$ of exposure.
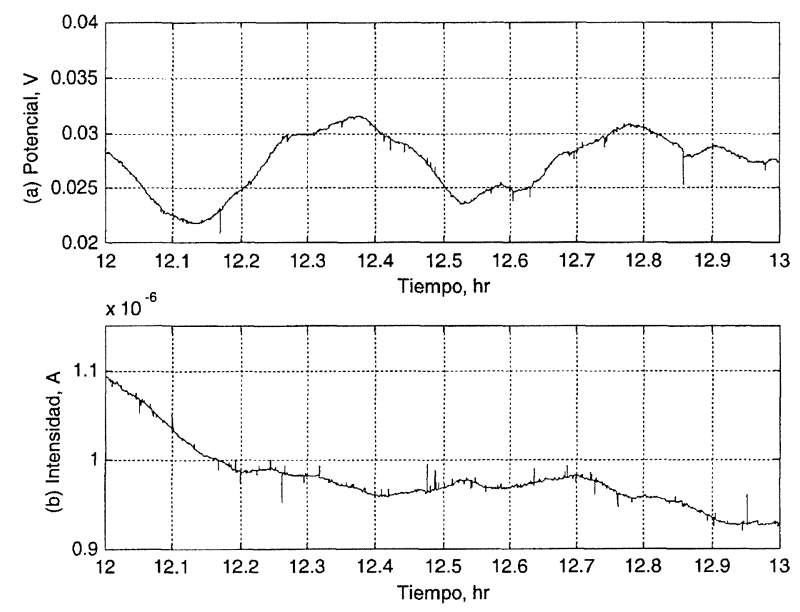

Figura 3. Registros de ruido electroquímico de potencial (a) e intensidad (b) del aluminio en $\mathrm{NaCl}$ al 3,5\% (picaduras) después de $12 \mathrm{~h}$ de exposición.

Figure 3. Electrochemical noise measurements for potential (a) and current (b) for aluminium in $\mathrm{NaCl}$ at $3.5 \%$ (pitting) after $12 \mathrm{~h}$ of exposure.

A simple vista pueden observarse ya diferencias importantes en los patrones de ruido electroquímico de potencial e intensidad en las tres condiciones. Los parámetros estadísticos de los registros se encuentran en la tabla II. Entre ellos cabe destacar los valores tan distintos de la resistencia de ruido, parámetro que en la bibliografía se contempla como uno de los más útiles a la hora de interpretar los datos de ruido electroquímico ${ }^{[9}$ y 10$]$. Así, en condiciones de pasivación la resistencia de ruido
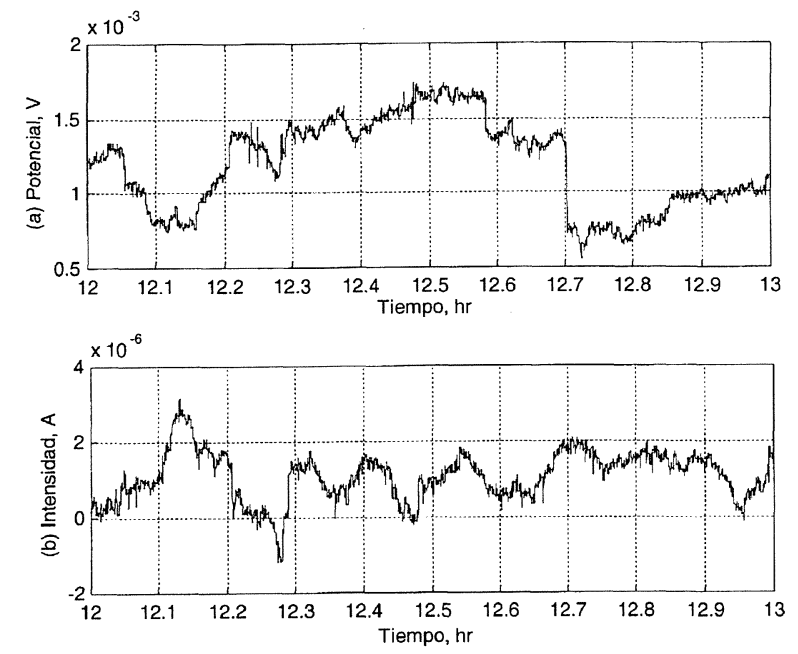

Figura 4. Registros de ruido electroquímico de potencial (a) e intensidad (b) del aluminio en $\mathrm{NaOH} 1 \mathrm{M}$ (corrosión generalizada) después de $12 \mathrm{~h}$ de exposición.

Figure 4. Electrochemical noise measurements for potential (a) and current (b) for aluminium in $\mathrm{NaOH} 1 \mathrm{M}$ ( generalised corrosion) after $12 \mathrm{~h}$ of exposure.

Tabla II. Datos estadísticos (desviaciones típicas y resistencia de ruido, $\sigma \mathrm{V} / \sigma \mathrm{ll}$ ) de los registros de potencial e intensidad del aluminio en la duodécima hora de exposición

Table II. Statistical data (typical deviations and noise resistance, $\sigma V / \sigma l)$ for the potential and current measurements of aluminium in the twelfth hour of exposure

\begin{tabular}{lccc}
\hline Electrolito & \multicolumn{2}{c}{ Desviación Típica } & $\begin{array}{c}\text { Resistencia } \\
\text { de Ruido }(\Omega)\end{array}$ \\
\cline { 1 - 3 } & Potencial (V) & Intensidad (A) & \\
\cline { 2 - 4 } $\mathrm{H}_{2} \mathrm{O}$ destilada & $6,0591 \cdot 10^{-4}$ & $7,0915 \cdot 10^{-12}$ & $8,5443 \cdot 10^{7}$ \\
$\mathrm{NaCl} 3,5 \%$ & $2,6000 \cdot 10^{-3}$ & $3,5979 \cdot 10^{-8}$ & $7,3248 \cdot 10^{4}$ \\
$\mathrm{NaOH} 1 \mathrm{M}$ & $3,0299 \cdot 10^{-4}$ & $6,3660 \cdot 10^{-7}$ & $4,7594 \cdot 10^{2}$
\end{tabular}

es muy grande, del orden de 108 ohmios, pues al no haber corrosión, las oscilaciones de potencial son de pequeña magnitud y las de corriente mucho menores, como puede apreciarse en los valores de la desviación típica, mientras que en condiciones de corrosión generalizada es muy pequeña, del orden de 102 ohmios, ya que aunque las oscilaciones de potencial son pequeñas (ver Tabla II), la corriente es relativamente grande debido a la disolución del metal. Cuando el aluminio sufre picaduras el valor es intermedio, aunque más próximo al de la pasivación $105 \Omega$, como es de esperar, pues en el proceso de picaduras se produce poca variación de 
la intensidad (el proceso ocurre en una pequeña porción de la superficie total) pero grandes saltos de potencial debido a la constante formación de pilas locales de corrosión.

Se observa que cuanto mayor es la resistencia de ruido, menor es la corrosión de un metal en el medio. Esta conclusión coincide con las que se pueden extraer de diversos estudios anteriores ${ }^{[11}$ y 12$]$.

\subsection{Análisis de Fourier}

Cualquier registro de datos en el dominio del tiempo puede ser representado, de modo alternativo, en el dominio de la frecuencia como la suma de cierto número de ondas sinusoidales de diferente amplitud. La integral de Fourier ${ }^{[8]}$ proporciona una función de la frecuencia que es la transformada de Fourier. Esta función es compleja: su módulo es la amplitud espectral y el cuadrado de la amplitud es la densidad de potencia espectral, DPE. Esta densidad espectral es, por otra parte, la transformada de Fourier de la autocorrelación. El término espectro se utiliza indistintamente para la amplitud y para la densidad de potencia representadas frente a la frecuencia.

La DPE puede determinarse por varios métodos. Los más empleados son el método de la máxima entropía y el cuadrado de la transformada de Fourier. Un ejemplo de los algoritmos utilizados en bibliografía para su cálculo, aparece en la ref. ${ }^{[13]}$. La DPE de los registros de intensidad y potencial parece ser un factor que suministra una información útil sobre el proceso que ocurre en los electrodos. Así, en diversos trabajos ${ }^{[1]}$ se menciona que la pendiente de la DPE de potencial está íntimamente relacionada con el tipo de corrosión (uniforme, pi- caduras, etc.). En la figuras 5a, 5b, 6a, 6b, 7a y $7 \mathrm{~b}$ pueden observarse las densidades de potencia espectral de los registros de ruido electroquímico correspondientes a las señales de las figuras 2,3 y 4 , tanto para potencial (a) como para intensidad (b).

En la tabla III se reflejan los valores de las pendientes de la densidad de potencia espectral y su magnitud para cada una de las señales (potencial e intensidad) de ruido electroquímico del aluminio en los tres medios estudiados.

Como puede observarse en la gráfica 5, la potencia espectral de potencial e intensidad, cuando el aluminio está pasivo, produce una señal plana y con frecuencias fundamentales, es decir, con cier-
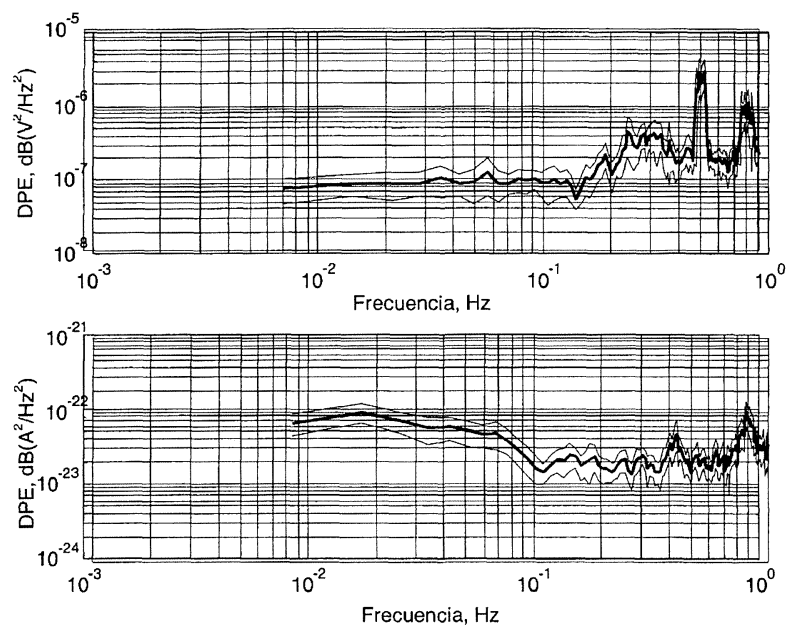

Figura 5. Densidad de potencia espectral: (a) potencial y (b) intensidad. Al en agua destilada (pasivación) después de 12 $\mathrm{h}$ de exposición.

Figure 5. Power spectral density: (a) potential and (b) current. Aluminium in distilled water (pasivation) after $12 \mathrm{~h}$ of exposure.

Tabla III. Pendientes de la densidad de potencia espectral y magnitud de la misma del ruido electroquímico del aluminio en las tres disoluciones empleadas al cabo de $12 \mathrm{~h}$ de exposición. La pendiente se expresa en decibelios por décadas de la magnitud expresada en las correspondientes unidades

Table III. Slopes of the power spectral density and PSD magnitude for electrochemical noise of aluminium in three solutions used after $12 \mathrm{~h}$ of exposure. The slope is expressed in decibels per decade for the magnitude expressed in the corresponding units

\begin{tabular}{lcccc}
\hline & \multicolumn{2}{c}{ Pendiente de la DEP } & \multicolumn{2}{c}{ Magnitud de la DEP } \\
\cline { 2 - 5 } Electrolito & $\begin{array}{c}\text { Potencial } \\
\mathrm{dB}\left(\mathrm{V}^{2} / \mathrm{Hz}^{2}\right) / \mathrm{dec}\end{array}$ & $\begin{array}{c}\text { Intensidad } \\
\mathrm{dB}\left(\mathrm{A}^{2} / \mathrm{Hz}^{2}\right) / \mathrm{dec}\end{array}$ & $\begin{array}{c}\text { Potencial } \\
\mathrm{dB}\left(\mathrm{V}^{2} / \mathrm{Hz}^{2}\right)\end{array}$ & $\begin{array}{c}\text { Intensidad } \\
\mathrm{dB}\left(\mathrm{A}^{2} / \mathrm{Hz}^{2}\right)\end{array}$ \\
\hline $\mathrm{H}_{2} \mathrm{O}$ destilada & 0 & -3 & $-60 \mathrm{a}-70$ & $-220 \mathrm{a}-230$ \\
$\mathrm{NaCl} 3,5 \%$ & -19 & -20 & $-60 \mathrm{a}-90$ & $-170 \mathrm{a}-180$ \\
$\mathrm{NaOH} 1 \mathrm{M}$ & -15 & -13 & $-70 \mathrm{a}-110$ & $-120 \mathrm{a}-160$ \\
\hline
\end{tabular}


Ventajas del análisis Wavelet sobre el análisis de Fourier para la interpretación del ruido electroquímico

L. Espada, M. Sanjurjo, S. Urréjola, F. Bouzada, G. Rey y A. Sánchez
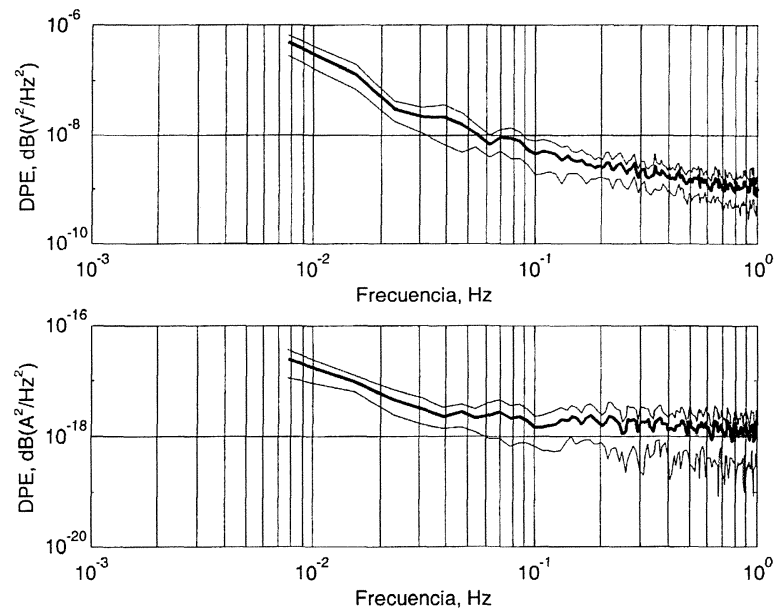

Figura 6. Densidad de potencia espectral: (a) potencial y (b) intensidad. Al en $\mathrm{NaCl}$ al 3,5\% (picaduras) después de 12 $\mathrm{h}$ de exposición.

Figure 6. Power spectral density: (a) potential and (b) current. Aluminium in $\mathrm{NaCl}$ at 3,5\% (pitting) after $12 \mathrm{~h}$ of exposure.
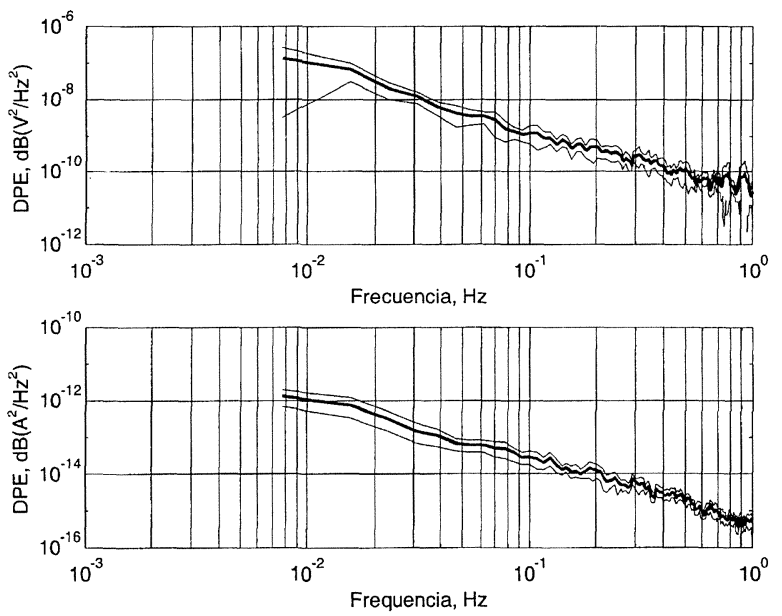

Figura 7. Densidad de potencia espectral: (a) potencial y (b) intensidad. Al en $\mathrm{NaOH} 1 \mathrm{M}$ (corrosión generalizada) después de $12 \mathrm{~h}$ de exposición.

Figure 7. Power spectral density: (a) potential and (b) current. Aluminium in $\mathrm{NaOH} 1 \mathrm{M}$ (generalised corrosion) after $12 \mathrm{~h}$ of exposure.

tos grados de periodicidad fácilmente identificables. Sin embargo, en los otros medios la potencia espectral obedece una ley $f^{-\alpha}$, en la que $f$ es la frecuencia, que es la que sigue la mayor parte de los registros de ruido electroquímico ${ }^{[12]}$. Sin embargo, la pendiente de la potencia espectral, $\alpha$, no es muy distinta en el caso de corrosión generalizada ( $\mathrm{Al}$ en $\mathrm{NaOH} 1 \mathrm{M}$ ) y en el de corrosión por picaduras ( $\mathrm{Al}$ en $\mathrm{NaCl}$ al 3,5 \%), ni en cuanto al caso del ruido de potencial ni al del ruido de intensidad.
Además, se observa que el orden de magnitudes de la pendiente de la potencia espectral de potencial e intensidad es el mismo en cada medio, lo que no refleja la relación, sugerida anteriormente por otros autores $^{[1]}$, de que la pendiente de la densidad de potencia espectral del ruido de potencial informa sobre el tipo de corrosión y que la pendiente de la densidad de potencia espectral del ruido de intensidad informa sobre la velocidad del proceso. Mejor que en este parámetro de la pendiente habría que fijarse en los valores de la magnitud de la propia potencia espectral de la intensidad (ver Tabla III) en los que sí queda claro que la magnitud de la potencia es indicadora de la velocidad de corrosión. Así, cuando el aluminio está pasivo, la magnitud de la potencia espectral de la intensidad es muy pequeña, mucho menor que cuando existe un proceso de picaduras, en cuyo caso es a su vez inferior que en el de corrosión generalizada.

Es importante resaltar que del análisis de Fourier no se extraen conclusiones mucho más importantes que del análisis estadístico de los registros de ruido electroquímico. Ello es debido, sin duda, a que esta metodología es útil casos de series de datos que presentan un cierto grado de periodicidad, como se aprecia en el caso del aluminio en agua destilada donde sí se pueden identificar frecuencias características del sistema, pero no en otros.

\subsection{Análisis de Wavelet}

La transformada de Fourier representa una herramienta útil para extraer la información contenida en una señal en el dominio de la frecuencia. Sin embargo, se ha demostrado ${ }^{[14]}$ que no sirve para la localización de eventos en el dominio del tiempo y la frecuencia simultáneamente. En este sentido, la transformada de Wavelet se muestra como un tratamiento matemático mucho más adecuado a la hora de extraer información de señales con pocos componentes de frecuencia fundamentales, como es el caso de la mayoría de las señales de ruido de potencial e intensidad que se pueden registrar en sistemas electroquímicos ${ }^{[7]}$.

La transformada de Wavelet se usa para analizar series temporales que contienen una potencia no estacionaria y no periódica. No es objeto este trabajo el profundizar en la metodología del análisis wavelet sino en su aplicación al ruido electroquímico, sin embargo se explicarán a continuación unos términos, a modo de introducción, que permitan seguir el desarrollo del trabajo. 
Supongamos una serie temporal, $x_{n}$, de espaciado temporal constante $\delta t$ y $n=0 . . \mathrm{N}-1$. Una función wavelet es una función que depende de un parámetro adimensional $\eta, \Psi_{0}(\eta)$. Para ser admisible como función Wavelet debe estar normalizada, es decir, debe tener valor medio nulo y estar localizada tanto en el tiempo como en la frecuencia ${ }^{[15]}$. El término "función Wavelet" se refiere a una función ortogonal o no ortogonal. El término "base Wavelet" se refiere a un conjunto de funciones ortogonales. El uso de una base de funciones ortogonales implica el empleo de la transformada Wavelet discreta, TWD, mientras que el empleo de funciones no ortogonales permite usar la transformada de wavelet continua, TWC, o la discreta, TWD. En este trabajo se emplea sólo la transformada continua, por lo que se ha elegido una función Wavelet no ortogonal (existen muchas familias de funciones wavelet).

La transformada Wavelet continua de una secuencia discreta, $x_{n}$, se define como la convolución de $\mathrm{x}_{\mathrm{n}}$ con una versión de la función Wavelet $\Psi_{0}(\eta)$ escalada en el espacio y trasladada en el tiempo:

$$
W_{n}(s)=\sum_{n^{\prime}=0}^{N-1} x_{n^{\prime}} \Psi *\left[\frac{\left(n^{\prime}-n\right) \delta t}{s}\right]
$$

donde (*) indica la compleja conjugada. Variando la escala Wavelet y trasladándola a lo largo de un índice $\mathrm{n}$ de la serie temporal, se puede construir una imagen de la amplitud o cualquier otra variable que muestre cómo cambia ésta frente a la escala con el tiempo.

Comentar que uno de los problemas más importantes que se presenta a la hora de aplicar el análisis Wavelet, y que ha sido achacado como una "arbitrariedad" de esta metodología, es la elección de la función Wavelet base, $\Psi_{0}(\eta)$, a utilizar en el análisis. En relación a esto, deben de considerarse varios factores que han suscitado la elección para el presente trabajo de una función Wavelet denominada "sombrero mexicano". En primer lugar, en este trabajo se utiliza la transformada Wavelet continua, por lo que se pueden usar funciones Wavelet no ortogonales. Otro factor a tener en cuenta es si la función es real o compleja: en el caso de series de número reales las funciones reales se adaptan mejor al análisis, mientras que las complejas lo hacen mejor a series complejas. La función Wavelet usada en los cálculos es:

$$
\Psi_{0}(\eta)=\frac{(-1)^{m+1}}{\sqrt{\Gamma\left(m+\frac{1}{2}\right)}} \frac{d^{m}}{d \eta^{m}}\left(e^{-\eta^{2} / 2}\right)
$$

donde $m=2$ y $\Gamma$ es el parámetro de normalización.

\subsection{Espectro de Potencia de Wavelet}

Por analogía con el análisis de Fourier, se puede definir el espectro de potencia Wavelet como el cuadrado de la transformada Wavelet, $|\mathrm{W}(\mathrm{s})|^{2}$. Si la función fuese compleja, su espectro de potencia Wavelet vendría dado por el cuadrado de la parte real de la transformada Wavelet. En este caso, la función "sombrero mexicano" es real y, por tanto, no tiene parte imaginaria.

Para hacer comparables espectros Wavelet de señales distintas, el espectro de potencia Wavelet se suele normalizar por un factor $\sigma^{2} / \mathrm{N}$, donde $\sigma^{2}$ es la varianza y $\mathrm{N}$ el número de puntos de la serie temporal. En las figuras $8 \mathrm{a}, 8 \mathrm{~b}, 9 \mathrm{a}, 9 \mathrm{~b}, 10 \mathrm{a}$ y $10 \mathrm{~b}$ se pueden observar los espectros de potencia Wavelet de las señales de ruido electroquímico del aluminio en los medios estudiados.

En este tipo de representación en el eje de abscisas se representa el tiempo de duración de la señal (en este caso una hora, la duodécima de exposición), en el de ordenadas los períodos (o su inversa, las frecuencias) y el valor de la potencia Wavelet se representa por áreas, tanto más oscuras cuanto mayor es su módulo. El valor de los períodos correspondería aproximadamente, aunque no de forma estricta ${ }^{[3]}$, a las frecuencias del espectro de Fourier. La línea negra gruesa representa el intervalo de confianza del $95 \%$ en el que el espectro se puede considerar fiable, es decir, en el que no se producen distorsiones por la propia transformada ${ }^{[5]}$.

Es importante resaltar la mayor información que se obtiene del espectro de potencia Wavelet respecto del obtenido con el análisis de Fourier. En él se puede apreciar tanto la duración de los picos y su ancho, como el cambio en el tiempo que la potencia de la señal va sufriendo, lo que conduce a espectros diferentes en función del tipo de corrosión. Así, cuando el metal está pasivo (Fig. 8) las oscilaciones de potencial e intensidad son regulares, de poca duración y pequeña distribución en el tiempo. En el caso de la intensidad, la simetría del espectro Wavelet denota claramente la existencia de frecuencias fundamentales, así como de una cierta reproducibilidad temporal de las varia- 
Ventajas del análisis Wavelet sobre el análisis de Fourier para la interpretación del ruido electroquímico L. Espada, M. Sanjurjo, S. Urréjola, F. Bouzada, G. Rey y A. Sánchez

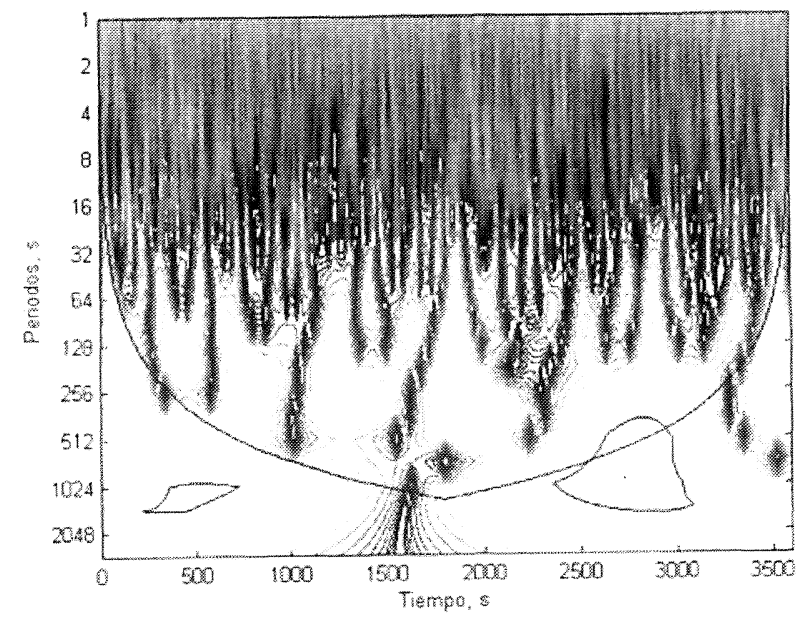

(a)

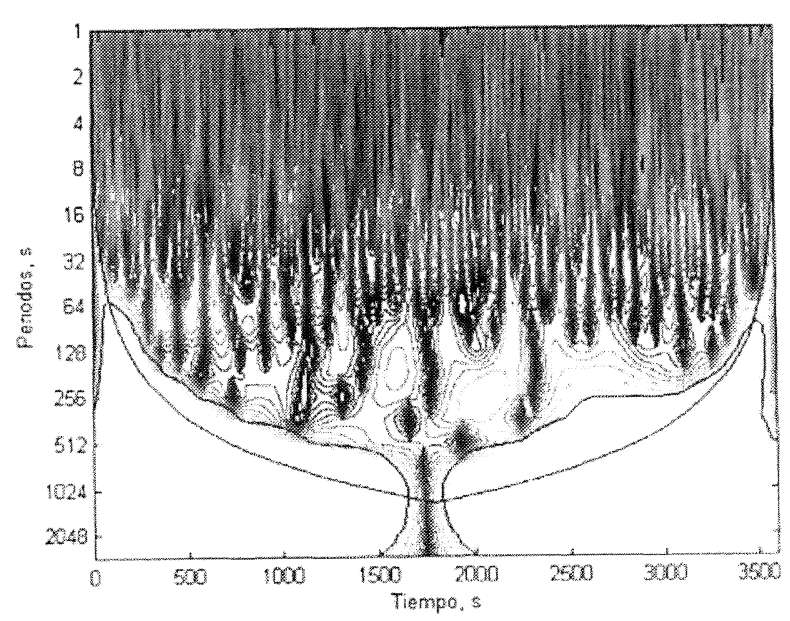

(b)

Figura 8. Espectro de potencia Wavelet: (a) potencial y (b) intensidad. Al en agua destilada (pasivación).

Figure 8. Wavelet power spectrum: (a) potential and (b) current. Aluminium in distilled water (pasivation).

ciones, hecho este indicativo de que la señal puede ser fractal o caótica.

En el caso de la corrosión por picaduras, se observan eventos de mayor relevancia y menos periodicidad, dando un espectro en el que la potencia se concentra en episodios de corta duración y frecuencia no periódica (espontáneos), como es de esperar por el tipo de proceso fisicoquímico de la formación de picaduras. Por último, en el caso de la corrosión generalizada volvemos a observar episodios de corta duración, muy amplia potencia y frecuencia alta, tanto en el caso del potencial, como de la intensidad.

Señalar que, como se observaba en el análisis de Fourier, el análisis Wavelet corrobora la afirmación anterior relativa a la similitud entre los espec-

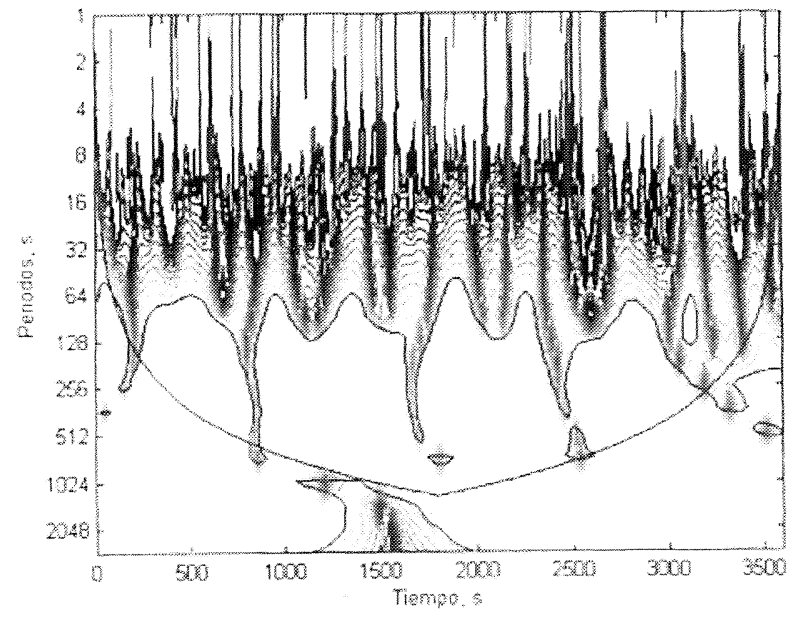

(a)

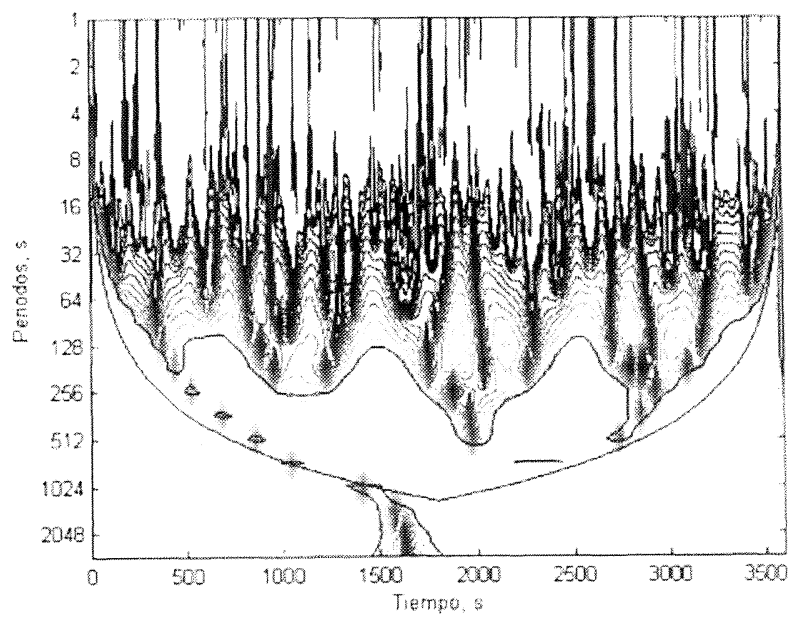

(b)

Figura 9. Espectro de potencia Wavelet: (a) potencial y (b) intensidad. Al en $\mathrm{NaCl}$ al 3,5\% (picaduras).

Figure 9. Wavelet power spectrum: (a) potential and (b) current. Aluminium in $3.5 \% \mathrm{NaCl}$ (pitting).

tros del potencial y la intensidad, es decir, la alta correlación que existe entre ambos y el hecho de que los registros de potencial e intensidad no informan de "cosas" distintas, como por otro lado era de esperar, pues la propia naturaleza del proceso electroquímico hace que los tránsitos de potencial y los de intensidad no sean eventos no relacionados, pues la disolución de un átomo metálico en el electrolito (salto de potencial) conlleva el paso de una cierta corriente eléctrica (salto de intensidad).

\section{CONCLUSIONES}

En este trabajo se muestra la aplicación de la metodología del análisis Wavelet a registros de ruido electroquímico de potencial e intensidad. Se sien- 


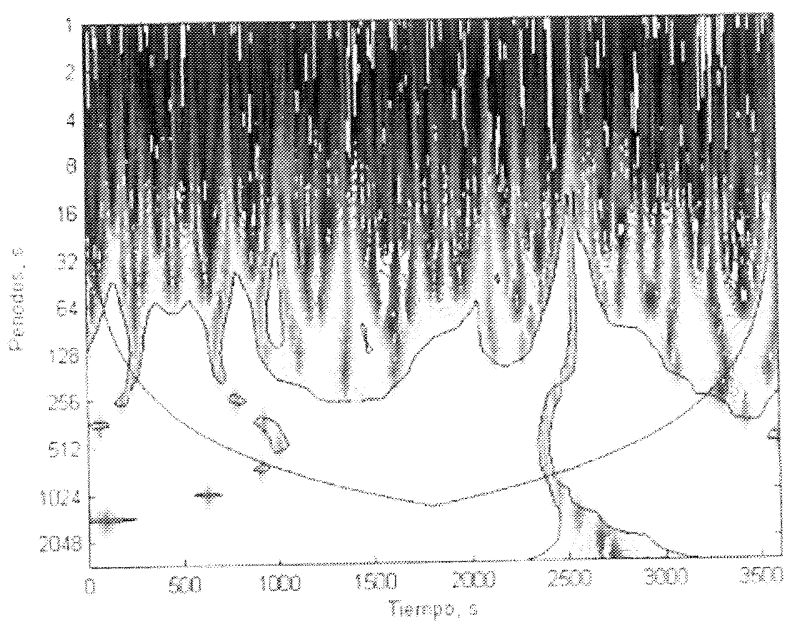

(a)

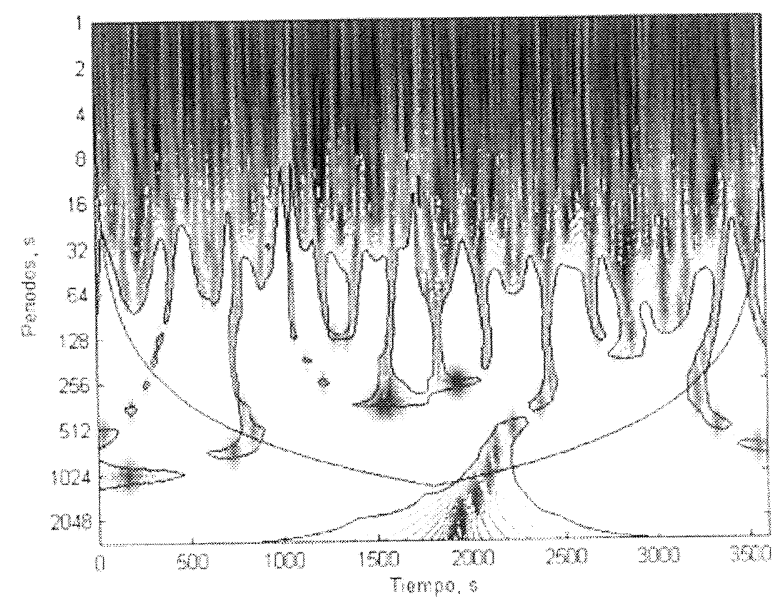

(b)

Figura 10. Espectro de potencia Wavelet: (a) potencial y (b) intensidad. Al en $\mathrm{NaOH} 1 \mathrm{M}$ (corrosión generalizada).

Figure 10. Wavelet power spectrum: (a) potential and (b) current. Aluminium in $\mathrm{NaOH} 1 \mathrm{M}$ (generalised corrosion).

tan las bases prácticas de la implementación de esta metodología al caso concreto de los registros de ruido electroquímico: series temporales reales, por lo que es necesario usar una función Wavelet real y no ortogonal.

También se demuestra mediante la técnica que, a pesar de lo que era admitido, los registros de potencial e intensidad no dan informaciones diferentes sino complementarias del proceso, aunque este hecho se puede deducir del análisis de Fourier.

Por último, se ofrece el análisis Wavelet como una alternativa mucho más precisa que el análisis de Fourier como vía para la obtención de información de los procesos que ocurren en el sistema electroquímico, pues se puede obtener información no solamente de la variación de los parámetros en el dominio de la frecuencia, sino su cambio durante todo el período de duración del experimento.

\section{Agradecimientos}

Este trabajo ha sido realizado, en parte, por la ayuda concedida al proyecto MAT1998-0537 de la Comisión Interministerial de Ciencia y Tecnología.

\section{REFERENCIAS}

[1] A. Legat y V. DOLECEK, J. Electrochem. Soc. 1426 (1995) 1.851.

[2] M. MOON y B. Skerry, J. Coat. Technol. 67843 (1995) 35.

[3] C. Torrence y P. Compo, Bull. Am. Meteo. Soc. 79 (1998) 61-78.

[4] H. Weng y K.M. LaU, J. Atmos. Sci. 51 (1994) 2.5232.541 .

[5] S.D. Meyers, B.G. Kelly y J.J. O'Brien, Mon. Wea. Rev. 121 (1993) 2.858-2.866.

[6] E. Foufoula-Georgiou, Wavelet in Geophysics, Academic Press, 1995, p. 373.

[7] I. DAubeCHIEs, IEEE Trans. Inform. Theory 36 (1990) 961-1.004.

[8] A. Oppenheim y R. SChafer, Discrete-time signal processing, cap. 8, 9 y12, Prentice-Hall Inc., 1989.

[9] F. Mansfeld, H. Xiao, L.T. Han, C.C. Lee, C. Chen, C. PÉREZ y J. Jones-MeEHAN, International Conference on Microbiologically influence Corrosion, 47, New Orleans, L.A., NACE, 1995.

[10] F. Mansfeld, L.T. Han y C.C. LeE, J. Electrochem. Soc. 12 (1996) 143, L286.

[11] B.S. Skerry y D.A. Eden, Progr. Org. Coat. 19 (1991) 379.

[12] L. Beaunier, J. Frydman, C. Gabrielli, F. Huet y M. KedDam, Electrochemical Noise Measurement, ASTM STP 1277, 1996, p. 114.

[13] E.O. Brigham, The Fast Fourier Transform, Prentice-Hall, Englewood Cliffs, N. J., 1974.

[14] G. KAISER, A friendly Guide to Wavelets, Birkhäuser, 1994, p. 300 .

[15] M. Farge, Annu. Rev. Fluid Mech. 24 (1992) 395-457. 